\title{
Monte Carlo ab initio simulation of the absorption spectrum of $\mathrm{Na}_{4}$
}

\author{
Romuald Poteau, Daniel Maynau and Fernand Spiegelmann \\ IRSAMC, Laboratoire de Physique Quantique (URA S05 du CNRS), Université Paul Sabatier, 118, route de Narbonne, \\ 31062 Toulouse Cedex, France
}

Received 1 February 1993; in final form 11 May 1993

\begin{abstract}
The influence of the classical vibration of the nuclei on the visible absorption spectrum of the $\mathrm{Na}_{4}$ cluster is investigated. The classical motion on the ground state potential energy surface at a given temperature is obtained from a Metropolis Monte Carlo simulation. This dynamical process makes possible a theoretical determination of the widths of the structures in the absorption spectra. The absorption spectrum is integrated from ab initio CI calculations of excited states and oscillator strengths along the random trajectory. A first simulation was achieved at $T=150 \mathrm{~K}$ using an ab initio $\mathrm{CI}$ determination of the ground and the excited states. The efficiency of the Metropolis process is improved with the use of an approximate representation for the ground state. Simulations are performed for several temperatures $T=50,150$, and $500 \mathrm{~K}$. The simulated spectra are discussed in relationship with the experimental spectrum of Kappes et al.
\end{abstract}

\section{Introduction}

The experimental determination of the geometrical structure of clusters from spectroscopic data is not straightforward. However, in the case of small alkali clusters, ESR techniques have been applied to the trimer [1] and the heptamer [2] trapped in argon matrices, and have indirectly shown the pentagonal symmetry of $\mathrm{Na}_{7}$. More recently, it has been shown that the study of photoabsorption spectra is also an attractive way to get information about the geometries. Photodepletion spectroscopy is the most currently applied technique to neutral or ionic clusters [3-5]. It allows one to access a wide range of excited states. Due to the probable dissociative character (directly or indirectly) of the excited states of alkali clusters for $n \geqslant 4$, most of the work using the laser induced fluorescence (LIF) [6] or the multiphoton ionisation (MPI) [7] techniques concerns trimers. In recent years, Bonacic-Koutecky, Fantucci and Koutecky (BKFK) have compared ab initio vertical spectra (calculated at the CI level) with depletion spectra in the case of small neutral or ionic alkali clusters $[1,8-13]$, and have underlined the correspondence between the nuclei positions and the electronic structure [14].

The link between geometrical structure and ab- sorption spectra is however not always straightforward. In order to point out the difficulties of the structural assignment of the depletion spectra, let us look at the results of BKFK for $\mathrm{Na}_{n}(n=5-8)$ [911]. The depletion spectra of $\mathrm{Na}_{5}$ and $\mathrm{Na}_{7}$ are assigned to the planar $C_{2 v}$ shape and the $D_{5 h}$ pentagonal bipyramid respectively [9]. In the case of $\mathrm{Na}_{6}$, three low energy isomers are in competition [9]. The lowest one is a pentagonal pyramid $\left(\mathrm{C}_{5 \mathrm{v}}\right)$, quasidegenerate with a planar $D_{3 h}$ geometry. The third structure has higher energy, and is built around a tetrahedron core. It has been shown to be the most stable isomer in the case of $\mathrm{Li}_{6}$. Although the experimental spectrum is close to the vertical one of the planar $D_{3 h}$ structure, the pentagonal pyramid leads to a theoretical spectrum which is not so different. The ambiguity is even more remarkable in the case of $\mathrm{Na}_{8}$. BKFK have considered three distinct structures $[10,11]$. The lowest one is a fully capped tetrahedron with $T_{d}$ symmetry, and has a near spherical shape, in agreement with the predictions of the jellium model. The two other structures are not minima at the Hartree-Fock (HF) level, their optimisation by a steepest descent algorithm yielding to the $T_{d}$ geometry. Nevertheless, they represent equilibrium structures in the framework of the density functional theory (DFT). The first one is a distorted piece of the fcc lattice $\left(D_{2 d}\right)$, 
and the second one is a square antiprism with $D_{4 d}$ symmetry. The $T_{d}$ and $D_{2 d}$ structures exhibit dominant features at $\approx 2.5 \mathrm{eV}$, while the most intense peak in the case of the $D_{4 d}$ geometry is located at $\approx 2.7 \mathrm{eV}$. However, the agreement of the calculated spectrum of the latter with the depletion spectrum is as good as for $T_{d}$ and $D_{2 d}$ structures.

One can notice that the broadening of the absorption bands is correlated to the interaction between electronic levels and the vibrational motion of the nuclei. We suggest that the ambiguity in the interpretation of spectroscopic data should be removed by taking into account the vibration of the nuclei around their equilibrium position. We have studied the influence of the nuclei motion in the ground state on the theoretical spectrum in the case of $\mathrm{Na}_{4}$ by the application of a Monte Carlo Metropolis algorithm.

\section{Computational aspects}

\subsection{Monte Carlo algorithm}

The motion of the nuclei on the ground state potential energy surface is treated via a Monte Carlo (MC) process [15]. The initial geometry is the $D_{2 h}$ rhombus in its equilibrium geometry (long diagonal $=12.4 \mathrm{bohr}$; short diagonal $=6.0 \mathrm{bohr}$ ). $\mathrm{A}$ randomly chosen atom $n$ is displaced with a small amplitude along one of the three principal axes $\left(\Delta q_{n}=0.1\right.$ bohr; $q_{n}=x_{n}$ or $y_{n}$ or $z_{n}$ also chosen randomly). The new configuration is accepted or rejected according to the Metropolis criterion [16]. The probability of jump from the initial configuration (with energy $E_{\delta}^{(i)}$ ) to the new one (of energy $E_{\delta}^{(i+1)}$ ) is given by ( 0 refers to the electronic ground state and $(i)$ to the MC iteration )

$$
\begin{array}{ll}
1, & \text { if } E_{\delta^{(i+1)}}-E_{\delta}^{(i)}<0, \\
\exp \left(-\frac{\left.E_{\delta}^{(i+1)}-E \delta^{(i)}\right)}{k_{\mathrm{B}} T}\right), & \text { if } E_{\delta^{(i+1)}}-E_{\delta}^{(i)}>0 .
\end{array}
$$

From a practical point of view, when $\left.E \delta^{(i+1)}\right)-E_{\delta}^{(i)}>0$, a random number $\nu$ is compared to $\exp \left(-\Delta E / k_{\mathrm{B}} T\right)$. The new configuration is accepted if $\nu<\exp \left(-\Delta E / k_{\mathrm{B}} T\right)$.

The use of classical dynamics (or equivalently of a
Metropolis sampling) instead of a quantum treatment for the vibrational motion is certainly questionable in the vicinity of the zero point vibration. In fact, we have calculated in a different work (using a monoelectronic Hamiltonian) the six harmonic frequencies of the rhombus, which are found to be 149.5, $134.9,98.6,94.5,77.1$, and $26.2 \mathrm{~cm}^{-1}$ respectively. Thus, the vibrational zero point energy is located at $290 \mathrm{~cm}^{-1}$ above the minimum of the potential energy surface. This energy corresponds to an estimate of the temperature (from the classical kinetic equivalence) of $\approx 35 \mathrm{~K}$ which may affect the results in the limit of low temperature. In any case, no classical simulation should be performed lower than $35 \mathrm{~K}$.

Whenever a configuration is accepted, the energies of the excited states are computed as well as the oscillator strengths corresponding to the optically allowed vertical excitations from the ground state to those excited states. The energies of the ground $\left(E_{\delta}^{(i+1)}\right)$ and excited states $\left(E_{m}^{(i+1)}\right)$ are given by an ab initio calculation (the computational details are given in section 2.2). A histogram is built from the integration of the transition energies $\epsilon_{m}^{(i)}=E_{m}^{(i)}-$ $E_{\delta}^{(i)}$ and the corresponding oscillator strengths $f_{m}^{(i)}$ along the random trajectory. The total energy range $\Delta=[0,3.5 \mathrm{eV}]$ is divided into small intervals $\Delta_{k}=$ $\left[\epsilon_{k}, \epsilon_{k}+\delta \epsilon\right.$ ] (with $\delta \epsilon=0.1 \mathrm{eV}$ ). If a transition energy $\epsilon_{m}^{(i)}$ belongs to $\Delta_{k}$, the corresponding oscillator strength $f_{m}^{(i)}$ is summed out in the interval. The intensity $I$ in a given interval $\Delta_{k}$ is given by

$$
\begin{aligned}
& f_{\mathrm{e}}\left(\Delta_{k}\right)=\sum_{i} \sum_{m} \frac{8 \pi^{2} \mu \epsilon_{m}^{(i)}}{3 h^{2} e^{2}}\left|\left\langle\psi \delta^{(i)}|R| \psi_{m}^{(i)}\right\rangle\right|^{2} \delta_{k, q_{i m}}, \\
& q_{i m}=\operatorname{int}\left(\frac{\epsilon_{m}^{(i)}}{\delta \epsilon}\right),
\end{aligned}
$$

where $e$ and $\mu$ are the charge and the mass of an electron respectively and $\delta$ is the Kronecker symbol.

After a few iterations, the plot of the histogram provides a temperature-dependent spectrum instead of vertical lines. In the following sections, histograms are plotted using linear interpolation.

\subsection{Ab initio calculation}

The effects of the core electrons are treated through the pseudopotentials of Durand and Barthelat [17], 
corrected by an $l$-dependent core-polarisation potential (CPP) [18].

In usual ab initio calculations, the molecular orbitals are traditionally constructed from the atomic orbitals (LCAO, linear combination of atomic orbitals) which are described with Gaussian functions. This yields to a linear increase of the basis with cluster size. This linear dependence may be reduced if one refers to the physical content of the spherical jellium model often used to describe metal clusters. Indeed, in this model, the nuclei are replaced by a uniform positively charged background. The resolution of the corresponding Hamiltonian yields sphericaltype orbitals [19]. We have taken our inspiration from these results in order to describe alkali clusters, and we have used a basis set (see table 1) centered on a fictitious site located at the center of mass of the cluster. Moreover, two s Gaussian functions are centered on each atom in order to properly account for the local contributions of the wavefunction. Notice that this kind of basis is relevant for geometries not too far from compactness.

Concerning the CPP correction, the core polarisability of sodium $\alpha$ is taken from ref. [20] ( $\alpha=0.995$ $a_{0}^{3}$ ). The cut-off radii have been determined in order to reproduce the energies of the lowest atomic states

Table 1

Exponents and coefficients of the Gaussian functions used in this work. The exponents for the sodium are taken from ref. [21]. The two $s$ functions result from a $(3,2)$ contraction

\begin{tabular}{llllll}
\hline \multirow{2}{*}{ Type } & \multicolumn{2}{l}{ Sodium } & & & \multicolumn{2}{l}{ Center of mass site } \\
\cline { 2 - 3 } \cline { 5 - 6 } & exponent & coeff. & & exponent & coeff. \\
\hline s & 2.835670 & 0.007012 & & 0.070 & 1 \\
& 0.493184 & -0.186763 & & 0.028 & 1 \\
& 0.072085 & 0.348672 & & 0.011 & 1 \\
& 0.039063 & 0.506590 & & 0.004 & 1 \\
& 0.019274 & 0.249458 & & \\
p & & & 0.056 & 1 \\
& & & 0.023 & 1 \\
& & & 0.009 & 1 \\
& & & 0.004 & 1 \\
d & & & 0.040 & 1 \\
& & & 0.016 & 1 \\
& & & 0.0065 & 1 \\
& & & 0.0025 & 1 \\
\hline
\end{tabular}

in each symmetry, namely $3 \mathrm{~s}, 3 \mathrm{p}, 3 \mathrm{~d}$. These radii are given in table 2.

The wavefunction is mainly built on singly excited determinants. Indeed, Bonacic-Koutecky et al. have shown that the transition energies and the associated oscillator strengths corresponding to the optically allowed electronic excitations from the ground state of the rhombus shape of $\mathrm{Na}_{4}$ can be, at least qualitatively, essentially described by linear combinations of monoelectronic excitations [8]. Nevertheless, we have verified that, in order to compute the correlation energy, a variational space which only contains single excitations does not yield satisfactory quantitative transition energies. We have checked with the MRCI-CIPSI method [22] that the inclusion of doubly excited determinants in the variational space improves the values of both transition energies and oscillator strengths. However, the calculation of the energy in a Monte Carlo process should be straightforward. This is not the case with the usual CIPSI method, where the variational space is built iteratively. It would indeed involve a search for the dominant determinants for each MC geometry, and would result in a rather complex procedure.

We have consequently used a more straightforward method. All the singly and doubly excited determinants with respect to the reference function (the HF wavefunction $\psi_{0}$ of the $\mathrm{Na}_{4}$ ground state) were treated variationally (SDCI). The total energy of a state $m$ was computed as the sum of the $m$ th root of the SDCI diagonalisation and the perturbative energy corresponding to the contribution of all the triple and quadruple excited determinants with respect to $\psi_{0}[23]$ :

$E_{m}=E_{m}^{\mathrm{SD}}+\sum_{K \in\{\mathrm{T}, \mathrm{Q}\}} \frac{\left|\left\langle\psi_{m}^{\mathrm{SD}}|H| K\right\rangle\right|^{2}}{\epsilon_{m}-\epsilon_{K}}$,

where S, D, T, Q, refer respectively to the singly, doubly, triply, and quadruply excited determinants and

Table 2

l-dependent cut-off radii of the core-polarisation potential $\left(a_{0}\right)$

\begin{tabular}{ll}
\hline$l$ & $\rho_{\mathrm{c}}$ \\
\hline 0 & 1.455 \\
1 & 1.600 \\
2 & 0.700 \\
\hline
\end{tabular}


$\psi_{m}^{\mathrm{SD}}=c_{m 0} \psi_{0}+\sum_{I \in\{\mathrm{S}, \mathrm{D}\}} c_{m I}|I\rangle$

This of course does not completely ensure an equivalent level of representation for the ground and excited states. A SDCI space generated from a multireference space including $\psi_{0}$ and all single substitutions should certainly be more appropriate, but far too CPU-time-consuming to be used in a simulation. The oscillator strengths were computed with the SDCI wavefunctions.

\subsection{Results for $\mathrm{Na}_{2}$}

Well-to-well transition energies and equilibrium distances for different states of the dimer obtained with the $(4 s 4 p 4 d+2 s / a t o m)$ basis set are listed in table 3 , and compared to other theoretical and experimental values. The equilibrium distance of the ground state $\left({ }^{1} \Sigma_{\mathrm{g}}^{+}\right)$is $5.95 \mathrm{bohr}$, slightly longer than the experimental value [24] ( $5.82 \mathrm{bohr})$. The dissociation energy $(0.68 \mathrm{eV})$ is smaller than the experimental value of Verma et al. [24] $(0.74 \mathrm{eV})$. BKFK found $0.703 \mathrm{eV}$ in an all-electron-MRDCI calculation with a 7s4pld per atom basis set also used for the calculation of the vertical spectrum of $\mathrm{Na}_{4}$. The largest error of our values with respect to the experimental ones when available is 0.13 bohr $\left(\mathrm{X}^{1} \Sigma_{\mathrm{z}}^{+}\right)$and $1100 \mathrm{~cm}^{-1}\left({ }^{1} \Pi_{\mathrm{u}}\right)$ in the case of the equilibrium distances and the transition energies respectively. With regard to the results of BKFK [8], these differences are $0.20 \mathrm{bohr}$ and $800 \mathrm{~cm}^{-1}$.

\section{Optical response of $\mathrm{Nan}$}

\subsection{Static spectrum}

We have also checked the quality of this $(4 s 4 p 4 d+2 s / a t o m)$ basis set for the determination of the vertical spectrum of the equilibrium structure of $\mathrm{Na}_{4}$ in the range $0-3.5 \mathrm{eV}$. We have checked the stability of the results with a more extended central basis set (6s6p6d3f - see table 4), and have compared both results with the experimental spectrum and the data of BKFK.

The measured spectrum was obtained by the group of Kappes [31]. They counted eight bands labelled from $A$ to H. BKFK have introduced three other labels, namely $E^{\prime}, F^{\prime}$, and $G^{\prime}$, which correspond to small experimental peaks. We have assigned two supplementary peaks, labelled E" and $F^{\prime \prime}$ (see fig. 1a).

The SCF configuration of the ground state of $\mathrm{Na}_{4}$ ( $\left.{ }^{1} A_{z}\right)$ is $\left(1 a_{g}^{2} 1 b_{3 u}^{2}\right)$. We have computed four excited states in each of the three allowed symmetries $\left({ }^{1} B_{1 u},{ }^{1} B_{2 u},{ }^{1} B_{3 u}\right)$. The results are given in table 5 . The excitation energies $T_{e}$ lie in the range $1.5-3.5 \mathrm{eV}$. It appears that $\mathrm{Na}_{4}$ is unsatisfactorily bound with respect to the dissociation into two dimers. Indeed, the dissociation energy is $0.17 \mathrm{eV}$, far from the value of BKFK $(0.41 \mathrm{eV})$. Nevertheless, as it was already the case for $\mathrm{Na}_{2}$, this default of our central basis set seems to have no influence on the transition energies (see table 5). The energies of the ground and excited states seem to be shifted by approximatively the same amount. The maximum error of our transition energies in view of the experimental ones is $0.12 \mathrm{eV}$, while it is $0.17 \mathrm{eV}$ in the AE-MRDCI calculation of BKFK [8].

Table 3

Equilibrium diotances $\left(a_{0}\right)$ and well-to-well transition energies $\left(10^{3} \mathrm{~cm}^{-1}\right)$ of some states of $\mathrm{Na}_{2}$

\begin{tabular}{|c|c|c|c|c|c|c|c|c|}
\hline \multirow[t]{2}{*}{ State } & \multicolumn{2}{|c|}{ This work } & \multicolumn{2}{|c|}{ SCF-CIPSI [25] } & \multicolumn{2}{|c|}{ SCF-MRDCI [8] } & \multicolumn{2}{|c|}{ Experiment } \\
\hline & $R_{0}$ & $T_{c}$ & $R_{e}$ & $T_{e}$ & $\boldsymbol{R}_{e}$ & $T_{\mathrm{e}}$ & $R_{0}$ & $T_{\mathrm{e}}$ \\
\hline $\mathrm{X}^{1} \mathbf{\Sigma}_{\mathbf{B}}^{+}$ & 5.95 & 0 & 5.80 & 0 & 6.025 & 0 & $5.82^{\star \prime}$ & 0 \\
\hline$x^{3} \Sigma_{u}^{+}$ & 9.95 & 5.34 & 10.00 & 5.9 & 9.746 & 5.49 & & \\
\hline$b^{3} \Sigma^{+}$ & 7.20 & 18.12 & 7.10 & 18.4 & 7.464 & 18.25 & & \\
\hline$A^{1} \Sigma+$ & 6.95 & 14.61 & 6.80 & 14.7 & 7.070 & 14.81 & $6.87^{b)}$ & $14.68^{c)}$ \\
\hline$a^{3} \Pi_{.}$ & 5.85 & 14.12 & 5.80 & 13.6 & 6.031 & 13.70 & & $13.65^{d)}$ \\
\hline $1 \Pi_{u}$ & 6.50 & 21.40 & 6.40 & 20.7 & 6.651 & 21.11 & $6.47^{\circ)}$ & $20.32^{n)}$ \\
\hline
\end{tabular}

a) Ref. [24]. () Ref. [26]. ") Ref. [27]. ') Ref. [28]. ") Ref. [29]. ' Ref. [30]. 
Table 4

Exponents of the Gaussian functions centered on the fictitious central site (6:6p6d3f)

\begin{tabular}{llll}
\hline s & p & d & f \\
\hline 0.070 & 0.056 & 0.085 & 0.056 \\
0.038 & 0.028 & 0.040 & 0.020 \\
0.017 & 0.014 & 0.017 & 0.008 \\
0.008 & 0.007 & 0.006 & \\
0.003 & 0.003 & 0.0025 & \\
0.0012 & 0.0012 & 0.0010 & \\
\hline
\end{tabular}

The vertical static spectra are plotted in fig. 1 . Our absorption features are roughly comparable to those of BKFK. Nevertheless, one can identify mainly two discrepancies between the latter and our spectrum obtained in the 4s4p4d basis set (fig. $1 \mathrm{~b}$ ):

(i) BKFK [8] have identified the transition towards 2 ' $\mathrm{B}_{2 \mathrm{u}}$ as the small peak $\mathrm{E}$ ' located at the bottom of the intense band $E$, leading to the fact that the latter should correspond to $\mathbf{3}^{\mathbf{}} \mathbf{B}_{2 u}$. Nevertheless, the shoulder of the $E$ band cannot be assigned. Moreover, in our work, the oscillator strength of the transition to $2{ }^{1} \mathrm{~B}_{2 \mathrm{u}}$ is larger than the one associated with $3^{1} B_{2 u}$. Our conclusion is that on one hand, the transition to $2{ }^{1} \mathrm{~B}_{2 \mathrm{w}}$ should be assigned to the $E$ band, and on the other hand, the excitation to the $3^{1} \mathrm{~B}_{2 u}$ corresponds to the shoulder of the $E$ band, that we labelled E".

(ii) the transition energy of the $3^{\prime} B_{3 u}$ obtained with the AE-MRDCI calculation ( $G^{\prime}$ peak) is $3.03 \mathrm{eV}$, and the corresponding oscillator strength is weak. In our case, this transition also has a weak oscillator strength, but its energy is $2.68 \mathrm{eV}$. Consequently, this band is located at the bottom of the $F$ band (we have labelled it $F^{\prime}$ instead of $G^{\prime}$ ).

Moreover, one can state that the transition energy to $3{ }^{1} \mathrm{~B}_{1 \mathrm{u}}$ obtained by BKFK [8] is weaker than the experimental value or than our value. The $E$ and $F$ bands have analogous oscillator strengths, while the experimental intensity of the $E$ band is larger than the intensity of the $F$ band.

We now compare the vertical spectrum obtained with the $(4 s 4 p 4 d+2 s / a t o m)$ basis set with the results obtained in the $(6 \mathrm{~s} 6 \mathrm{p} 6 \mathrm{~d} 3 \mathrm{f}+2 \mathrm{~s} /$ atom $)$ central basis set (fig. lc). There are four differences.

(i) The $\mathrm{C}$ band is less intense in the large basis set
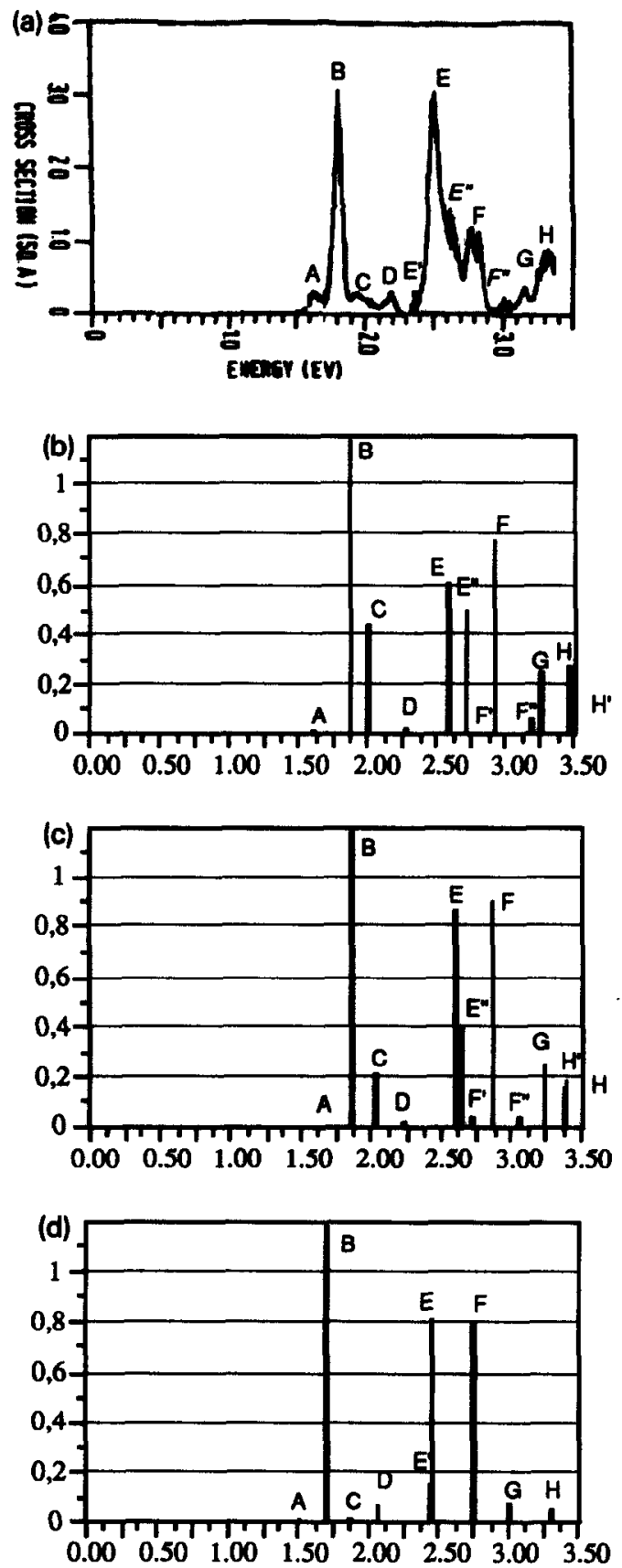

Fig. 1. (a) experimental spectrum of $\mathrm{Na}_{4}$ (after Wang et al. [31]); (b) ab initio (pecudopotential) vertical spectrum obtained in a (2s/atom + 4s4p4d at the center of mass) basis set; (c) ab initio (paeudopotential) vertical spectrum obtained in a (2s/atom + 6s6p6d3f at the center of mass) basis set; (d) ab initio (all electron) vertical spectrum in a 7s4p1d/atom basis set [8-14]. 
Table 5

Transition energies (in eV) and associated oscillator strengths for Na. The oscillator strengths are normalised with respeet to the one of the B peaks of BKFK [8]. The experimental transition energies are located in the table in such a way that they correspond to the assignment made in this work

\begin{tabular}{|c|c|c|c|c|c|c|c|c|}
\hline \multirow[t]{2}{*}{ State } & \multicolumn{2}{|c|}{$4 s 4 p 4 d+2$ s/atom } & \multicolumn{2}{|c|}{$6 s 6 p 6 d 3 f+2 s / a t o m$} & \multicolumn{2}{|c|}{$7 \mathrm{~s} 4 \mathrm{p} 1 \mathrm{~d} /$ atom $[8,14]$} & \multicolumn{2}{|c|}{ Experiment [31] } \\
\hline & $T_{e}$ & $f_{e}$ & $T_{e}$ & $f_{e}$ & $T_{e}$ & $f_{0}$ & $T_{\mathrm{e}}$ & $\sigma\left(A^{2}\right)$ \\
\hline${ }^{1} B_{2 u}(1)$ & $1.60 \mathrm{~A}$ & 0.005 & $1.59 \mathrm{~A}$ & 0.0005 & $1.51 \mathrm{~A}$ & 0.008 & $1.63 \mathrm{~A}$ & 0.089 \\
\hline${ }^{1} B_{3 u}(1)$ & $1.86 \mathrm{~B}$ & 1.180 & $1.85 \mathrm{~B}$ & 1.180 & $1.71 \mathrm{~B}$ & 1.180 & $1.60 \mathrm{~B}$ & 1.180 \\
\hline${ }^{1} \mathbf{B}_{\mathbf{3 u}}$ (2) & $2.00 \mathrm{C}$ & 0.446 & $2.03 \mathrm{C}$ & 0.208 & $1.87 \mathrm{C}$ & 0.011 & $1.98 \mathrm{C}$ & 0.069 \\
\hline${ }^{1} B_{1 u}(1)$ & $2.27 \mathrm{D}$ & 0.014 & $2.22 \mathrm{D}$ & 0.019 & $2.07 \mathrm{D}$ & 0.075 & $2.18 \mathrm{D}$ & 0.097 \\
\hline${ }^{1} \mathbf{B}_{2 u}(2)$ & $2.57 \mathrm{E}$ & 0.601 & $2.61 \mathrm{E}$ & 0.867 & $2.45 \mathrm{E}^{\prime}$ & 0.150 & $2.51 \mathrm{E}$ & 1.168 \\
\hline${ }^{\prime} B_{3 u}(3)$ & $2.68 \mathrm{~F}^{\prime}$ & 0.0002 & $2.72 \mathrm{~F}^{\prime}$ & 0.038 & $3.03 \mathrm{G}^{\prime}$ & 0.002 & & \\
\hline${ }^{1} \mathbf{B}_{2 u}$ (3) & $2.71 \mathrm{E}^{\prime \prime}$ & 0.500 & $2.64 \mathrm{E}^{\prime \prime}$ & 0.387 & $2.46 \mathrm{E}$ & 0.810 & 2.63 & 0.365 \\
\hline \multirow{2}{*}{${ }^{1} B_{1 u}(2)$} & $2.91 \mathrm{~F}$ & 0.772 & $2.86 \mathrm{~F}$ & 0.905 & $2.76 \mathrm{~F}$ & 0.796 & $2.78 \mathrm{~F}$ & 0.324 \\
\hline & & & & & & & 2.85 & 0.304 \\
\hline${ }^{1} \mathbf{B}_{3 v}(4)$ & $3.17 \mathrm{~F}^{\prime \prime}$ & 0.057 & $3.06 \mathrm{~F}^{\prime \prime}$ & 0.039 & $3.00 \mathrm{G}$ & & $\approx 3.05$ & \\
\hline${ }^{1} B_{1 v}(3)$ & $3.26 \mathrm{G}$ & 0.247 & $3.24 \mathrm{G}$ & 0.240 & $3.30 \mathrm{H}$ & 0.083 & $3.15 \mathrm{G}$ & 0.081 \\
\hline${ }^{1} \mathbf{B}_{2 \mathrm{u}}(4)$ & $3.45 \mathrm{H}$ & 0.267 & $3.39 \mathrm{H}$ & 0.152 & & 0.056 & $3.33 \mathrm{H}$ & 0.341 \\
\hline${ }^{1} B_{\mathrm{lu}}(4)$ & $3.49 \mathrm{H}^{\prime}$ & 0.142 & $3.38 \mathrm{H}^{\prime}$ & 0.180 & & & & \\
\hline
\end{tabular}

(perhaps luckily), in better agreement with experiment.

(ii) The $E$ and $E^{\prime \prime}$ bands are more or less close as a function of the basis, yielding a different relative position of the small peak $F^{\prime}$.

(iii) The oscillator strengths of the $E$ and $F$ bands are bigger in the extended basis set, relative to the most intense peak (B).

(iv) There is an inversion of the quasidegenerate $H$ and $H^{\prime}$ bands. This could be explained by a better description of the highest excited states in the more extended basis.

\subsection{Ab initio dynamical spectrum at $T=150 \mathrm{~K}$}

The calculation of the dynamical spectrum of $\mathrm{Na}_{4}$ involves an increased computational effort. Indeed, the symmetry of the initial $D_{2 h}$ structure is lost, due to the distortions of the cluster. The calculation is consequently made in the $C_{1}$ symmetry group. Furthermore, there are numerous excited states in a quite narrow energy range. Despite the limitation of the calculation to singlet states only, the determination of the transition energies in the range $0-3.5 \mathrm{eV}$ involves the calculation of 25 singlets. The spectrum is plotted as a function of the number of iterations in fig. 2. After 20 iterations, the spectrum is still very close to the spectrum of the equilibrium structure. The
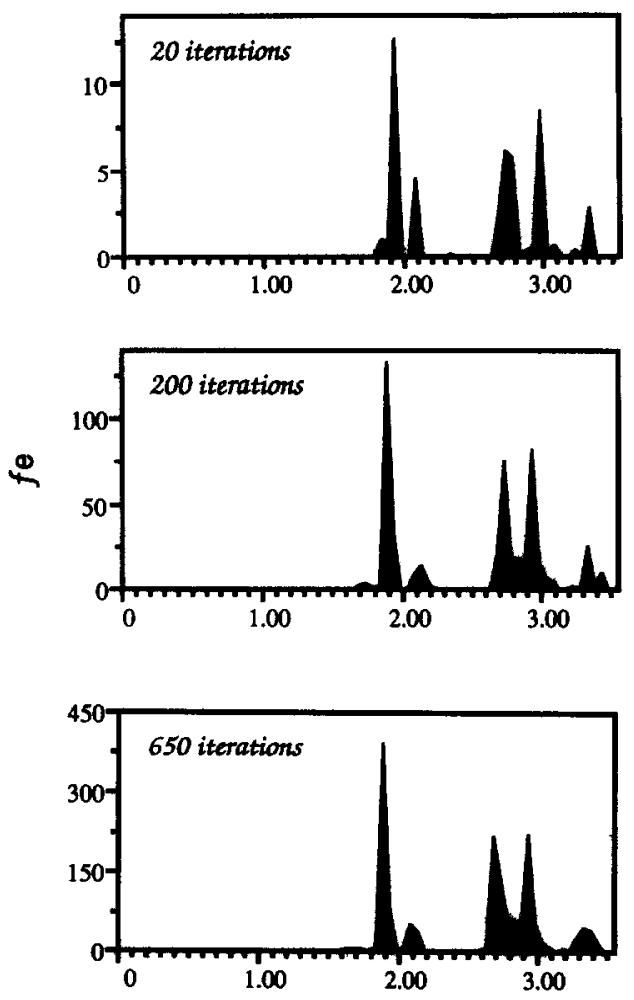

Fig. 2. Plots of the dynamical spectrum for various steps of the simulation. 
B peak is the most intense, and it is difficult to distinguish from the $E$ and $E^{\prime \prime}$ structures. The $F$ band is more intense than the $E$ band. As for the $C$ band, it is too intense, due to the basis set. At the 200th iteration, many modifications of the spectrum are noticeable. The B peak is still sharp and intense. On the other hand, the $E$ and $F$ bands have now similar intensities, and the sum of the oscillator strengths of the $E^{\prime \prime}$ and $F^{\prime}$ transitions provide the observed shoulder of the $E$ band. The $C$ band is becoming wider, but also less intense. Beyond approximatively the 400th MC iteration, the convergency of the spectrum is nearly reached, and we considered that the plot converged at the 650 th iteration. Indeed, we observed no modification of the shape of the spectrum between 400 and 650 iterations (apart from a global increase of the intensity). Of course, due to the time cost of the calculation, we were not able to perform further checks of convergence using ab initio calculations of the energy. The $E$ and $F$ bands have equivalent heights, which is also the case with the static spectrum of BKFK [8]. The $\mathbf{H}$ band is wide, and has merged with a small peak appearing at $\approx 3.5 \mathrm{eV}$. The intensity of structure $D$ in the final simulation is vanishing. A small peak at the bottom of the $E$ band has appeared. There is a satisfactory overall agreement between the dynamical and the experimental spectra [31], in particular for what concerns the widths of the $B, E$ and $F$ bands or the heights of the $B, C, F$, and $H$ bands. Nevertheless, $a$ few discrepancies are noticeable, mainly the underestimation of the intensity of the $E$ band, the overestimation of the $F$ band, and also the fact that we do not observe a splitting of the F structure.

\subsection{Approximate treatment of the ground state}

Despite the progress in computer technology, such an ab initio CI Monte Carlo process remains costly. Moreover, it is not certain that following the Monte Carlo trajectory over 650 geometries is sufficient from a statistical point of view. In particular, in order to avoid statistical correlation (the geometry of the cluster between two consecutive MC steps remains nearly identical), one often uses an algorithm where the properties are only accumulated for some of the $\mathrm{MC}$ steps (for instance every $50 \mathrm{MC}$ generations) This requires a much larger number of computations for the ground state. With the aim of treating larger clusters, one also wishes to reduce the time required for the calculation of the simulation. We have introduced in a previous paper a model Hamiltonian developed for sodium clusters [32]. It is an extension of a distance-dependent tight-binding monoelectronic Hamiltonian based on valence 3 s orbitals, including perturbatively the effect of $3 p$ orbitals. The computational effort to obtain the ground state energy is considerably reduced, since the energy is obtained as the sum of $n / 2$ eigenvalues of a $n \times n$ matrix. We have extensively checked its validity for small clusters $(n=2-9)$, from both a geometrical and an energetic point of view, and used it in order to study the geometrical and energetics properties of larger clusters $(n<34)$ [33]. Such a Hamiltonian has proved relevant for the description of the ground state potential energy surfaces of sodium clusters.

The number of randomly generated geometries was here taken as 4000 , from which $1 / 50$ were explicitly considered in the integration of the spectrum. From a practical point of view, the initial geometry is the equilibrium structure obtained with the model. Nevertheless, the bond lengths of the equilibrium geometries provided by the model are slightly shorter than those of the ab initio geometry. We have empirically taken into account that difference in the following way:

- all the modifications of the geometry which have been accepted according to the Metropolis criterion are stored;

- every 50 MC steps, these successive modifications are applied to the ab initio geometry, after multiplication by an empirical constant:

$\Delta q_{\mathrm{ab}}=1.1 \Delta q_{\text {model }}$.

The spectrum provided by the application of this algorithm is compared to the purely ab initio simulation for the same temperature $(T=150 \mathrm{~K})$. The peaks are located around the same transition energies, and have analogous heights (see fig. 3a). However, the width of most of them is slightly accentuated in this simulation (in particular B, C, E, and F). There are two possible explanations. On the one hand, the proportionality factor between the ab initio and model Hamiltonian coordinates is empiric; on the other hand, the potential energy surface curvatures provided by the two Hamiltonians are probably differ- 

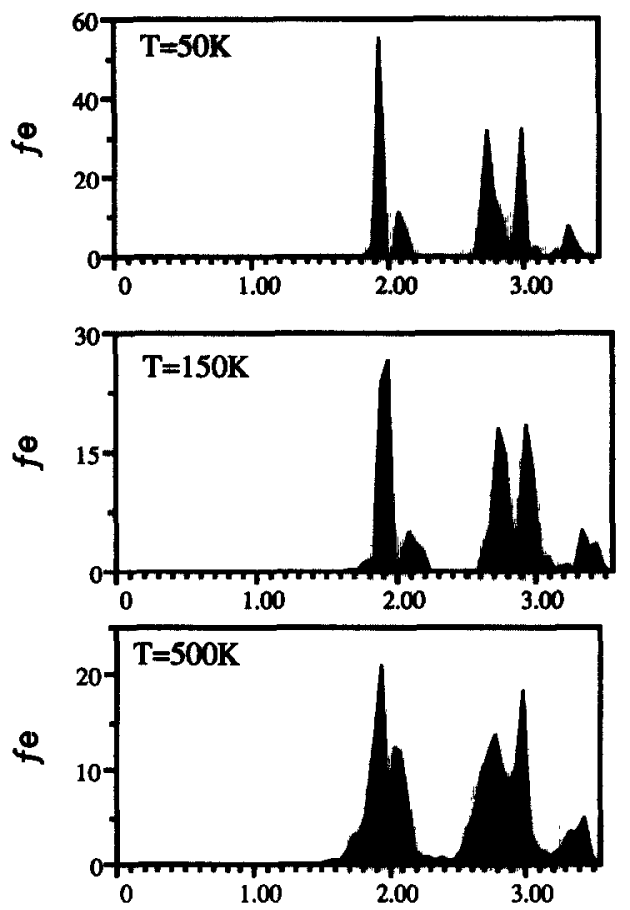

Fig 3. Influence of the temperature on the dynamical spectrum of Na.

ent. However, the overall agreement of the spectra is good.

\subsection{Influence of the temperature on the dynamical spectrum}

Due to the low cost of this simulation with respect to the ab initio MC simulations, it has been carried out for two other temperatures, namely $T=50 \mathrm{~K}$ and $T=500 \mathrm{~K}$ (see figs. $3 \mathrm{~b}$ and $3 \mathrm{c}$ ). The $T=50 \mathrm{~K}$ and $T=150 \mathrm{~K}$ spectra exhibit very close behaviours. The only noticeable difference lies in the widths of the bands, less important for $T=50 \mathrm{~K}$. However, the agreement with the experimental data is good, and it is not easy to discriminate between the two temperatures. On the other hand, the $T=500 \mathrm{~K}$ simulation provides a dynamical spectrum which is very different. As we have observed in a previous paper [34] in which we studied phase transitions in small sodium clusters $(n=4,8$, and 20$)$, at this temperature, the cluster becomes fluxional, and oscillates between the rhombus and the $T$ shape. The bands are very wide, in particular the $\mathrm{E}$ band, and the structure corresponding to the initially labelled $A, B$, and $C$ bands. The $C$ band is now very intense.

\section{Conclusion}

The use of ab initio methods for the study of the excited states of alkali clusters is a step towards the understanding and the analysis of experimental $a b-$ sorption spectra. The correspondence between the position of nuclei and electronic transitions may sometimes allow for the assignment of the experimental features with the geometry of the clusters. Nevertheless, the link between structure and vertical theoretical spectrum may be ambiguous (e.g. $\mathrm{Na}_{6}$ and $\mathrm{Na}_{8}$ ). In order to improve the description of theoretical spectra and provide a determination of the widths, we have taken into account the influence of the temperature on the nuclei motion by a Monte Carlo SCF-CI treatment. The application of this procedure for different temperatures yields an estimation of the temperature of sodium clusters in molecular beams. Of course, this thermometer cannot yet be considered as very accurate. Our conclusion is that the temperature of $\mathrm{Na}_{4}$ in the experiment of Wang et al. is rather low, probably between 50 and $150 \mathrm{~K}$.

The atomic basis set was built by considering the assumptions of the jellium model. An extended basis set (4s4p4d) is located at the center of mass of the cluster, leading to a reduction of the total number of atomic orbitals as the size of cluster increases. The ground state energy is unsatisfactorily reproduced, but the agreement of the transition energies with the experimental values is good.

It is also interesting to notice that the coupling of this process with our model Hamiltonian considerably reduces the computation time and yields results comparable with the purely ab initio process. This agreement is another indication of the relevance of our model for the description of small sodium clusters' potential energy surface.

\section{References}

[1] D.A Garland and D.M. Lindsay, J. Chem Phys. 78 (1983) 2813. 
[2] D.A. Garland and D.M. Lindsay, J. Chem Phys. 80 (1984) 4671.

[3] W.A. de Heer, K. Selby, V. Kresin, J. Masui, M. Vollmer, A. Chatelain, and W.D. Knight, Phys. Rev. Letters 59 (1987) 1805.

[4] C. Brechignac, Ph. Cahuzac, F. Carlier and J. Leygnier, Chem. Phys. Letters 164 (1989) 433.

[5] C. Wang, S. Pollack and M.M. Kappes, J. Chem. Phys. 93 (1990) 3867.

[6] J. Gole, G. Green, S. Pace and D. Preuss, J. Chem. Phys. 76 (1982) 2247.

[7] A. Herrmann, A. Hofmann, E. Schumacher and L. Wöste, Chem. Phys. Letters 62 (1979) 216.

[8] V. Bonacic-Koutecky, P. Fantucci and J. Koutecky, Chem. Phys. Lett. 166 (1990) 32.

[9] V. Bonacic-Koutecky, J. Pittner, C. Scheuch, M.F. Guest and J. Koutecky, J. Chem. Phys. 96 (1992) 7938.

[10] V. Bonacic-Koutecky, M.M. Kappes, P. Fantucci and J, Koutecky, Chem. Phys. Letters 170 (1990) 26.

[11] V. Bonacic-Koutecky, P. Fantucci and J. Koutecky, J. Chem. Phys. 93 (1990) 3802.

[12] V. Bonacic-Koutecky, P. Fantucci and J. Koutecky, Chem. Phys. Letters 146 (1988) 518.

[13] Ph. Dugourd, J. Blanc, V. Bonacic-Koutecky, M. Broyer, J. Chevaleyre, J. Koutecky, J. Pittner, J.P. Wolf and L. Wöste, Phys. Rev. Letters 67 (1991) 2638.

[14] V. Bonacic-Koutecky, P. Fantucci and J. Koutecky, Chem. Rev. 91 (1991) 1035.

[15] S. Kirkpatrick, C.D. Gelatt and M.P. Vecchi, Science 220 (1983) 671 .

[16] N. Metropolis, A. Rosenbluth, M. Rosenbluth, A. Teller and E. Teller, J. Chem. Phys. 21 (1953) 1087.

[17] Ph. Durand and J.C. Barthelat, Theoret. Chim. Acta 38 (1975) 283;

J.C. Barthelat, Ph. Durand, and A. Serafini, Mol. Phys. 33 (1979) 179;
J.C. Barthelat and Ph. Durand, Gazz. Chim. Ital. 108 (1978) 255.

[18] M. Foucrault, Ph. Millié and J.P. Daudey, J. Chem. Phys. 96 (1992) 1257.

[19] W. Knight, K. Clemenger, W.A. de Heer, W.A. Saunders, M.Y. Chou and M.L. Cohen, Phys. Rev. Letters 52 (1984) 2141.

[20] W. Müller, J. Flesch and W. Meyer, J. Chem. Phys. 80 (1984) 3297.

[21] D. Pavolini and F. Spiegelmann, J. Chem. Phys. 87 (1987) 2854.

[22] B. Huron, P. Rancurel and J.P. Malrieu, J. Chem. Phys. 58 (1973) 5475;

S. Evangelisti, J.P. Daudey and J.P. Malrieu, Chem. Phys. 75 (1983) 91.

[23] D. Maynau and J.L. Heully, to be published.

[24] K.K. Verma, J.T. Bahns, AR Rejei-Rizi, W.C. Stwalley and W.T. Zemke, J. Chem. Phys. 78 (1983) 3599.

[25] G. Jeung, J. Phys. B 16 (1983) 4289.

[26] M.E. Kaminsky, J. Chem. Phys. 66(1977) 4591.

[27] P. Kusch and M.M. Hessel, J. Chem. Phys. 63 (1975) 4087.

[28] B. Atkinson, J. Becker and W. Demtröder, Chem. Phys. Letters 87 ( 1982 ) 92.

[29] W. Demtröder and M. Stock, J. Mol. Spectry. 61 (1976) 382.

[30] F. Barrow, N. Travis and C.V. Wright, Nature 187 (1960) 141.

[31 ] C.RC. Wang, S. Pollack, D. Cameron and M.M. Kappes, J. Chem. Phys. 93 (1990) 3787.

[32] R. Poteau and F. Spiegelmann, Phys. Rev. B 45 (1992) 1878.

[33] R. Poteau and F. Spiegelmann, J. Chem. Phys. 98 (1993) 6540.

[34] R. Poteau, P. Labastie, and F. Spiegelmann, Z. Physik. D, submitted for publication. 